\title{
The nose is hungrier than the eyes
}

\author{
Karina Chan ${ }^{1} \cdot$ Richard J. Stevenson ${ }^{1} \cdot$ Heather Francis ${ }^{1}$
}

Accepted: 31 October 2020 / Published online: 17 November 2020

(C) The Psychonomic Society, Inc. 2020

\begin{abstract}
Vision and olfaction are the main sensory channels for appraising food prior to eating. Motivational models often assume that these sensory channels function in an equivalent manner. We tested this notion by asking participants to rate their desire for some snacks only via smell and others only via vision. In the next phase, participants consumed a small sample of every snack, now with all of the senses available, rating liking and desire for more. After consuming a meal, participants repeated the desire/liking test. Sensing via olfaction, relative to vision, led to greater desire ratings irrespective of state. When hungry, judgments of liking and desire for more were higher for foods that were initially smelled relative to those that were initially seen. Across the meal, visually based desire ratings declined more than those based on smell, relative to ratings made when the snacks were tasted. Together, this suggests motivational equivalence does not hold for olfaction and vision. We suggest this may be due to a greater reliance on memory for generating visually based desire.
\end{abstract}

Keywords Desire $\cdot$ Motivation $\cdot$ Olfaction $\cdot$ Pleasure

Decisions about whether or not to eat rely on two main sensory channels_-vision and olfaction (Stevenson, 2009). Food is seen in advertisements, on packaging, and on the plate, and it is smelled when passing a bakery or restaurant, during cooking, and when it is served. These senses, alone or in combination, can result in desire, with this moderated both by physiological state (i.e., hungry vs. sated) and by many psychological variables (e.g., dieting, food preferences; e.g., Hofmann \& Van Dillen, 2012; Kavanagh, Andrade, \& May, 2005). Desire and its moderating variables have been studied in some detail (e.g., Hofmann \& Van Dillen, 2012). This is because appetitive control seems easily overwhelmed by the modern food environment, with its frequent encounters with palatable food, often resulting in overeating, weight gain, and obesity (e.g., Redden \& Haws, 2013). There is, however, a key part of food desire that we know little about — namely, the role of each sensory channel in its formation. Two related assumptions are often made in this regard. First, that these channels are motivationally equivalent, with each being as

Richard J. Stevenson

dick.stevenson@mq.edu.au

1 Department of Psychology, Macquarie University, Sydney, NSW2109, Australia good as the other in generating desire (e.g., Jones et al., 2018; Kavanagh et al., 2005). Second, that desire resulting from different sensory channels is similarly moderated by physiological state (e.g., Berridge, 1996; Cabanac, 1992). That is hunger/fullness should exert the same effect on food desire, irrespective of the sensory channel from which it arises. Our aim is to test whether these related assumptions are correct.

If, for a given stimulus, smelling produced more pleasure than looking, then it seems reasonable to infer that smelling might also lead to greater desire. Thus, if there were normally differences in how affect-laden (i.e., pleasure/displeasure; Russell, 2003) smell and vision were, this should result in motivational differences, too. Many scientists have regarded a stimulus perceived via the olfactory channel as being more affect-laden than the same stimulus perceived via vision. For example, Wrzesniewski, McCauley, and Rozin (1999), comment that "for humans, it seems that the sense of smell (along with taste) stand out as having more immediate and direct emotional impact" (p.713), a view endorsed by several other researchers (e.g., Engen, 1982; Ehrlichman \& Bastone, 1992; Richardson \& Zucco, 1989; Rouby \& Bensafi, 2002; Smeets $\&$ Dijksterhuis, 2014; Zatorre, 2002). One reason for this broad support comes from the memory literature, with odorinduced memories being consistently more affect-laden than vision or audition-induced ones (e.g., Herz, 2004; Richardson 
\& Zucco, 1989). However, our focus here is on perception, and whether the same stimulus perceived via olfaction evokes a greater affective response than via vision. Several findings bear on this more specific question. Categorization of odorants is based upon affect (e.g., Dubois, 2000), odorants activate affect-related brain structures more so than pictures or sounds (Royet et al., 2000), and the same stimulus presented via olfaction evokes more affect-laden descriptors than when presented visually (Hinton \& Henley, 1993). These findings suggest that the same object will be perceived as more pleasant (or unpleasant) via olfaction than vision, and thus perhaps more (or less) desirable, too.

\section{ORDER \\ 1st

Judge four snacks: A, B, C, D
Experimenter provides name
Smell snack you can't see
Judge desire
Repeat

2nd
Test order counterbalanced

Snacks counterbalanced $\leftarrow \leftarrow \leftarrow \leftarrow \leftarrow \leftarrow \leftarrow \leftarrow \leftarrow \leftarrow \leftarrow \leftarrow \leftarrow \leftarrow$ $\rightarrow \rightarrow \rightarrow \rightarrow \rightarrow \rightarrow \rightarrow \rightarrow \rightarrow \rightarrow \rightarrow \rightarrow \rightarrow+1$

$\downarrow$

$\downarrow$

$\downarrow$

\section{VISUAL DESIRE}

Judge four snacks: E, F, G, H Experimenter provides name Look at snack you can't smell Judge desire Repeat

\section{TASTING PHASE}

$$
\begin{aligned}
& \text { For all eight snacks: A-H } \\
& \text { Experimenter provides name } \\
& \text { All snacks visible and uncovered } \\
& \text { Eat a single small bit of snack } \\
& \text { Judge liking } \\
& \text { Judge desire for more } \\
& \text { Repeat } \\
& \downarrow \\
& \downarrow
\end{aligned}
$$
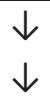

3rd

MEAL

Eat a filling meal

4th

OLFACTORY DESIRE

\begin{tabular}{|c|c|}
\hline Test order counterbalanced & VISUAL DESIRE \\
\hline Snacks counterbalanced & Judge four snacks: $E, F, G, H$ \\
\hline$\leftarrow \leftarrow \leftarrow \leftarrow \leftarrow \leftarrow \leftarrow \leftarrow$ & Experimenter provides name \\
\hline 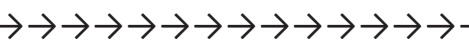 & Look at snack you can't smell \\
\hline$\downarrow$ & Judge desire \\
\hline$\downarrow$ & Repeat \\
\hline
\end{tabular}

Judge four snacks: $A, B, C, D$ Experimenter provides name Smell snack you can't see Judge desire Repeat

5th$$
\downarrow
$$

TASTING PHASE

$$
\begin{aligned}
& \text { For all eight snacks: } \mathrm{A}-\mathrm{H} \\
& \text { Experimenter provides name } \\
& \text { All snacks visible and uncovered } \\
& \text { Eat a single small bit of snack } \\
& \text { Judge liking } \\
& \text { Judge desire for more } \\
& \text { Repeat }
\end{aligned}
$$

Diagram 1 Summary of the experimental procedure, noting that hunger/fullness ratings were taken at various points during the procedure 
Unfortunately, the claim that olfaction is more affect-laden than vision is less robust than it appears. Odor categorization in the laboratory may depend upon affect because identification is poor without contextual cues (e.g., Cain, 1979). Many studies do not directly match olfactory, visual, and auditory stimuli, and thus different stimuli confound cross-modal comparison. Indeed, even the strongest test (Hinton \& Henley, 1993), which strictly matched stimuli, is ambiguous. Average stimulus-induced affect did not differ. Rather, olfactory presentation generated more extreme affective responses, either no emotion at all or lots of it, relative to vision. So, while there are grounds to suspect that for a given stimulus olfactory experience will be more affect-laden than visual experience- which, in turn, would suggest motivational differences, too- the evidence is equivocal.

As to whether visual and olfactory derived food desire are equally moderated by physiological state is even less researched. Ingestive alliesthesia occurs when a food-related stimulus evokes more pleasure when a small sample is eaten when hungry than when sated (Cabanac, 1979). Ingestive alliesthesia has been demonstrated for olfaction, with food smells rated as being more pleasant when hungry than when full (e.g., Duclaux, Feisthauer, \& Cabanac, 1973). Only one study has directly contrasted olfactory and visually presented food cues when hungry and when sated. When hungry, Jiang et al. (2008) found that participants' liking and desire ratings for colour food pictures were higher than for foods they could only smell. In addition, visual-based alliesthesia involved a larger change in desire and liking than olfactory-based alliesthesia. These results are difficult to interpret because of stimulus matching across modalities. Olfactory stimuli were composed of unlabelled artificial scents (e.g., pizza odorant) and the visual condition of colour food pictures matched to the odor name (e.g., slice of pizza). However, as odor identification is poor (e.g., pizza odorant may not always be identified as pizza odor), whether participants actually perceived them as matching is unknown.

Here, we examined whether fully matched and named (to avoid misidentification) sets of palatable snack foods, experienced via olfaction and vision, would (1) generate different degrees of desire and (2) be equally affected by a change in physiological state (i.e., eating a meal). We addressed this using a desire (also known as 'wanting' in the motivation literature) and liking test (Attuquayefio et al., 2016; Pender, Stevenson, Francis, \& Oaten, 2019; Stevenson et al., 2020; Stevenson, Francis, Attuquayefio, \& Ockert, 2017), with our design summarized in Diagram 1. Our approach assesses (1) desire from only looking at and from only smelling the snacks and then (2), after actually eating a small portion of each snack, liking and desire for more. These latter eating-based ratings were included to test for well-documented effects of ingestive alliesthesia (Cabanac, 1979), but more importantly to contrast with participants purely visual and olfactory based desire ratings. This procedure was then repeated after a filling meal.

\section{Method}

\section{Participants}

Thirty-six participants were required for this study to ensure full counterbalancing of the palatable snack foods used in this fully within-participant design (see Diagram 1). The study was advertised with the following inclusion and exclusion criteria, and those who did not meet them were asked to self-exclude. Exclusion criteria were as follows: age $<17$ or $>35$, a previous/current eating-related disorder, diabetic, food allergies, or other disorder affecting diet/eating or smell. Inclusion criteria were currently healthy, ages $17-35$, English as primary language, and no recent or current upper respiratory tract infection. In total 39 participants were run to fill the 36 counterbalanced cells of the design. Three were excluded during testing due to their failure to meet the inclusion/exclusion criteria - this being checked during the experiment (two were identified as obese [i.e., BMI >30] and one reported having had an eating disorder). Using their data rather than their replacements has no effect on the reported pattern of outcomes.

The protocol was approved by the Macquarie University Human Research Ethics Committee, and participants provided written consent to take part. The study aim was described only in general terms as concerning affective reactions to food. The specific study aims were provided in a debriefing at the end of the experiment. No participant demonstrated awareness of the specific study aims when asked its purpose prior to debriefing.

\section{Snack food stimuli}

The same eight snack foods were used on each desire and liking test. They were composed of four savoury and four sweet snacks, with the following amount of food used in each case: one Cheezel (cheese flavored extruded corn snack; Snack brands, Australia); one $0.5 \mathrm{~cm}^{3}$ piece of cheddar cheese (Mainland); one barbeque-flavored Pringles brand potato chip; one salt and vinegar flavored Pringles brand potato chip; one raspberry flavored frog-shaped jelly (Allen); one mini Tim-Tam chocolate covered and filled biscuit (Arnotts); one mini chocolate chip cookie (Arnotts); and one mini Bueno (Kinder; chocolate wafer snack).

\section{Counterbalancing of the snack food stimuli}

There were two desire and liking tests, one conducted when hungry and another when sated. Each desire and liking test was composed of two phases. In the first, participants judged 
desire for the eight snack foods, which were organized into two modality conditions where the stimuli were either just smelled or just viewed. Each modality condition was composed of four snack foods - two sweet and two savoury. Each sweet and savoury snack served equally often in the smelling and viewing modality conditions across the 36 participants (i.e., overall, each snack occurred 18 times in each modality) as well as occurring equally often with each other snack in each modality condition. The order of presentation of the snacks within each modality condition was randomized separately for every participant, with the same random order being used both before and after the meal to preserve judgmental context. The order in which the modality condition took place was counterbalanced across subjects, so that half always completed smelling before viewing, and the other half always completed viewing before smelling. For the second phase (tasting), the foods were presented in the same order for each participant as was used in the desire judgmentsagain, to maintain judgmental context.

\section{Procedure}

All participants were asked to breakfast (or lunch) as per nor$\mathrm{mal}$, and then refrain from eating in the 3 hours before testing so as to arrive hungry for lunch (i.e., arriving between 11 a.m. and 1:30 p.m.) or dinner (i.e., arriving between 4 p.m. and 6 p.m.). All participants reported complying with these provisions.

Participants started by completing biographical questions (native language, age, gender, alterations to dietary patterns [i.e., dieting], general health, presence of upper respiratory infections, injuries to nose or face or head, presence of metabolic or eating disorders). They were then asked to rate how hungry, full, thirsty, happy, sad, alert, and relaxed they were on 120-mm line rating scales (anchors not at all to very).

The two-phase desire and liking test was then completed. The first phase, desire judgments, was split into a separate viewing and smelling condition, which were undertaken in counterbalanced order. As order had no significant effect on outcome, this variable is not reported further.

For both the visual and olfactory based desire judgments, the snack foods were verbally identified by the experimenter as the participant looked at or smelled the snack. The rationale for this approach is important, as olfactory identification is often poor (e.g., Cain, 1979) and misidentification can significantly alter hedonic judgments (e.g., Herz \& von Clef, 2001). Thus, any comparison between these modalities without equating for identification is problematic because the participant may believe they are smelling a different odor to the one the experimenter believes they are presenting. Moreover, misidentification may occur idiosyncratically, if a name is not presented.
In the viewing condition, each participant was asked to look at four snack foods (two sweet, two savoury) in the specified order, with each placed in a sealed clear plastic jar (i.e., to eliminate any odor cue). For each snack, the participant inspected the sample in the jar, the experimenter said its name, and the participant rated how much they wanted to eat it now, using a $120-\mathrm{mm}$ line rating scale (anchors not at all and a lot).

In the smelling condition, each participant was asked to smell the other four snack foods (two sweet, two savoury; i.e., those not used in the viewing condition) in the specified order. Under the guidance of the experimenter, participants were asked to close their eyes and sniff the crushed-up snack in an opaque back plastic jar, again with the experimenter saying its name. After replacing the lid, participants were asked to open their eyes and rate how much they wanted to eat it now, using the same scale as for the viewing part. Finally, participants judged, overall, how clearly they could smell the four snack foods $(120-\mathrm{mm}$ line rating scale, anchors not at all and very).

The second phase of the desire and liking test, tasting, followed immediately. Here, participants received a new batch of the same eight snacks used in the previous phase, in the same order, this time with them all visible and uncovered on a tray. The task for each food was identical. They were asked to eat the whole portion of the small sample while the experimenter said its name, and then make two ratings, both on 120mm line rating scales: (1) How much do you like this food? (anchors not at all and $a$ lot); and (2) How much more of this food would you like to eat now? (anchors none and a lot). Following a water rinse, participants then repeated this process for each of the remaining snack foods.

Participants then evaluated how hungry, full, thirsty, happy, sad, alert, and relaxed they were, using the same method as described earlier. This was followed by lunch/dinner, which consisted of either $260 \mathrm{~g}$ of beef or vegetarian lasagna as chosen by the participant. They were asked to eat until they were comfortably full, with the added instruction that all unused food would be thrown away and that they were free to ask for more if still hungry. Ten minutes was given to consume lunch/dinner with ad libitum access to water. Participants were allowed to read magazines (content screened to avoid any eating-related or upsetting material), but were requested to leave all electronic devices with the experimenter. Amount eaten and drunk were recorded at the end of the study after the participant had left.

After completing a final set of state and mood scales (i.e., how hungry, full, thirsty, happy, sad, alert, and relaxed), the desire and liking test was repeated in an identical manner as described for the before lunch/dinner version. Participants were then asked to complete the Three Factor Eating questionnaire to assess dietary restraint, and were weighed and had their height and waist circumference measured. A debriefing 
regarding the study aims followed and this concluded the experiment.

\section{Analysis}

Data were suitable for parametric testing based upon Shapiro-Wilk testing and examination of descriptive statistics (i.e., means, standard deviations, skewness, kurtosis). For the premeal desire and liking test, and consistent with prior analyses of this test, ratings were collapsed across the four snacks that were initially judged for desire in the visual modality, and likewise for the four snacks initially judged for desire in the olfactory modality. This yielded a set of six premeal ratings (i.e., for initially vision-judged snacks - desire [on just seeing], liking and desire more [on tasting]; for initially olfaction-judged snacks - desire [on just smelling], liking and desire more [on tasting]). The same approach was adopted for the six postmeal ratings (for initially vision-judged snacksdesire [on just seeing], liking and desire more [on tasting]; for initially olfaction-judged snacks-desire [on just smelling], liking and desire more [on tasting]). An omnibus test was then used to analyze all 12 ratings, because it enabled tests of whether desire is influenced by the modality in which it is made (vision vs. olfaction), how modality influences changes in desire by physiological state (pre vs. post meal), and how all of this compares to liking and desire more ratings, made for the same snacks (i.e., those initially vision-judged vs. those initially olfactionjudged). This was operationalized in a state (hungry vs. sated) $\times$ rating type (desire vs. liking vs. want more $) \times$ modality (initially olfaction vs. initially vision), repeatedmeasures analysis of variance (ANOVA).

Hunger and fullness data are reported in brief format, with just their $p$ values and effect sizes, to confirm that the expected change in physiological state (hungry to full) occurred. Analyses of mood and thirst data were not undertaken, as these ratings were included to disguise our primary interest in the hunger and fullness evaluations.

Table 1 Hunger and fullness data obtained across the experiment using line rating scales

\begin{tabular}{lll}
\hline Time & Hunger & Fullness \\
\hline Before lunch & & \\
$\quad$ Before first desire and liking test & $68.5(24.7)$ & $33.0(24.9)$ \\
$\quad$ After first desire and liking test & $53.8(27.3)$ & $55.6(24.6)$ \\
After lunch & & \\
Before second desire and liking test & $12.9(11.7)$ & $100.1(14.6)$ \\
After second desire and liking test & $11.6(17.8)$ & $101.7(26.7)$ \\
\hline
\end{tabular}

\section{Results}

\section{Biographical information}

Participants had a mean age of 19.2 years $(S D=3.3)$ and a mean BMI of $21.7(S D=2.0)$. They reported being able to smell the snack foods on both the first $(M=105.4 / 120[S D=$ 15.3]) and second $(M=103.5 / 120[S D=18.8])$ desire and liking test, with only $3 / 72$ ratings less than the midpoint of this scale, suggesting participants could readily perceive these smells. Restraint scores were comparable to previous samples in our laboratory (e.g., Attuquayefio et al., 2016; Pender et al., 2019; Stevenson et al., 2020; Stevenson et al., 2017; restraint $M=7.8[S D=3.9])$.

\section{Hunger, fullness, and the meal}

Participants consumed an average of $225 \mathrm{~g}(S D=64 \mathrm{~g})$ of lasagna at lunch/dinner. Hunger and fullness ratings are presented in Table 1. Analysis of these data (separate two-way repeated-measures ANOVAs for hunger and for fullness ratings, with time of rating [before vs. after meal], time of test [before vs. after desire and liking test]) revealed that hunger significantly decreased and fullness increased across lunch ( $p$ s $<.001 ; \eta_{\mathrm{p}}{ }^{2} \mathrm{~s}>0.83$ ), indicating that the manipulation of state was successful. Changes in hunger and fullness also occurred across the desire and liking tests $\left(p \mathrm{~s}<.002 ; \eta_{\mathrm{p}}{ }^{2} \mathrm{~s}>0.24\right)$, but these were bigger before lunch than after $\left(p \mathrm{~s}<.003 ; \eta_{\mathrm{p}}{ }^{2} \mathrm{~s}>\right.$ 0.23).

\section{Comparing olfaction and vision on the desire and liking test}

The desire, liking, and desire more rating scores (see Fig. 1) were analyzed using a three-way repeated-measures ANOVA (state $\times$ rating type $\times$ modality). The ANOVA revealed a main effect of state, $F(1,35)=75.50, M S E=90.19, p<.001, \eta_{\mathrm{p}}{ }^{2}=$ 0.68 , with all ratings higher before eating than after (see Fig. $1)$. There was also a main effect of modality, $F(1,35)=6.07$, $M S E=85.57, p<.02, \eta_{\mathrm{p}}{ }^{2}=0.15$, with all ratings for foods that were initially smelled higher than all ratings for food that were initially viewed. In addition, there was a main effect of rating type, $F(2,70)=55.95, M S E=45.67, p<.001, \eta_{\mathrm{p}}{ }^{2}=0.62$, with both desire and want more ratings, overall, lower than liking ratings.

The main effects were qualified by interactions between state and rating type, $F(2,70)=16.70, M S E=16.78, p<$ $.001, \eta_{\mathrm{p}}{ }^{2}=0.32$, and by the three-way interaction of state, rating type, and modality, $F(2,70)=3.96, M S E=11.46, p<$ $.025, \eta_{\mathrm{p}}{ }^{2}=0.10$. As is evident in Fig. 1 , and concordant with the latter effect, the interaction between state and rating type was larger for vision, $F(2,70)=20.03, p<.001, \eta_{\mathrm{p}}{ }^{2}=0.36$, than it was for olfaction, $F(2,70)=4.99, p<.01, \eta_{\mathrm{p}}{ }^{2}=0.13$. 

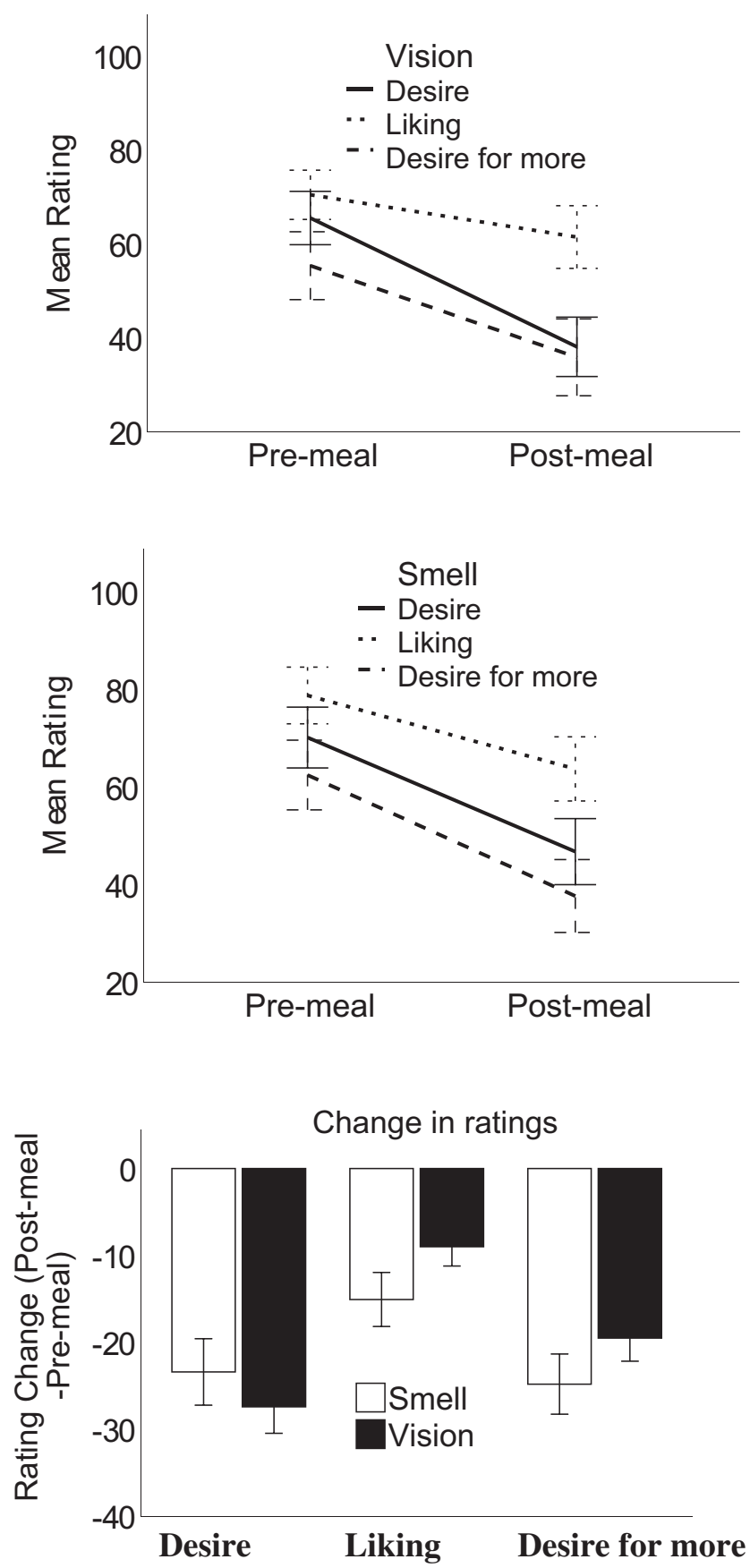

Fig. 1 Mean (and $S E$ ) desire ratings made when either looking at (top panel) or smelling (middle panel) the snack foods, and ratings of liking and desire for more, made when the food was seen and then consumed for foods initially seen (top panel) and smelled (middle panel). These evaluations were obtained before and after a filling meal, with the change in each rating type shown by modality in the lower panel

To identify why the interaction effect was bigger for vision, we contrasted the change across state in each rating type (i.e., desire vs. liking, desire vs. desire more, and desire more vs. liking) between vision and olfaction. Desire declined more across state relative to liking for vision $(M=-18.5)$ than it did for olfaction $(M=-8.4), t(36)=2.27, p=.029$. Desire declined more across state relative to desire more for vision $(M$
$=-8.0)$ than for olfaction $(M=1.4), t(36)=2.26, p=.03$. Change across state in liking relative to desire more judgments did not differ by modality $(t<1)$. In sum, solely visual-based desire decreased significantly more across state - relative to actual eating-based judgements - than solely olfactory-based desire.

Finally, we examined the premeal and postmeal data separately to determine whether modality exerted any general effect. For premeal ratings, the main effect of modality was significant, $F(1,35)=6.27, p<.02, \eta_{\mathrm{p}}^{2}=0.15$, with no interaction with rating type $(F<1, p=.53)$-so, here, all ratings were higher for snacks that were initially smelled. For postmeal ratings, the Modality $\times$ Rating Type interaction was significant, $F(2,70)=3.42, p<.05, \eta_{\mathrm{p}}^{2}=0.09$, so, in this case, modality-based effects depended upon the type of rating being made. We examined this by comparing each rating type by modality on the postmeal test. The only significant difference was for desire ratings, which were significantly higher for olfaction $(M=46.9)$ than for vision $(M=38.2), t(36)=$ $3.23, \mathrm{p}=0.003$.

\section{Discussion}

Vision and olfaction are the two main channels for perceiving food before it is eaten. This study examined whether these two sensory channels are motivationally equivalent —namely, are they equally effective at generating food desire, and are they equally affected by a change in physiological state. When hungry, snack foods that were initially smelled (vs. seen) had higher ratings of desire, liking, and desire for more. This is particularly notable for the liking and desire for more ratings, which were completed later after tasting the snacks. When sated, smelling the snacks led to elevated desire ratings relative to just seeing them, but this time it did not affect liking and desire for more ratings. Following the change in physiological state from hungry to sated, olfactory and visually based desire were differentially affected, relative to food-based judgments. Visually based desire decreased far more across state, relative to the actual food-based ratings, than did olfactory-based desire. Together, these data suggest that for the same set of palatable snack foods, (1) olfactorybased desire is reported as stronger (i.e., hungrier) than visually based desire, and (2) these sensory channels do not respond in the same manner to a shift in physiological state, as smell remains hungrier. Thus, the assumption of motivational equivalence does not always hold.

Before turning to the implications of this study, it is important to acknowledge potential limitations. First, formal olfactory testing to establish normosmia was not undertaken, which may have increased the variability among the olfactory-based responses. Nonetheless, participants reported being able to clearly smell the snack foods, and none reported any medical history suggestive of a deficit (e.g., injury to face). Second, the 
change in state manipulation was completed with a fixed test order (i.e., hungry then full) to enable testing in one session. Although it is not immediately apparent how this might adversely influence the outcome, another study ran the hungry and sated components of the desire and liking test on different days in counterbalanced order and found no significant effect of order (Zuniga et al., 2020). Third, the snack foods included sweet and savoury items, but the meal was just savoury, which might differentially influence ratings of each item type (i.e., sensory specific satiety; Rolls, Rolls, Rowe, \& Sweeney, 1981). Although this would not affect our conclusions for the vision versus olfaction comparisons, as each used two sweet and two savoury snacks, prior work that directly tested the effects of similarity between the intervening meal and the snacks on the desire and liking test, found no evidence of impact (Stevenson et al., 2017). Finally, we did not assess individual differences in attention to the olfactory domain (e.g., Croy, Buschhuter, Seo, Negoias, \& Hummel, 2010; Smeets, Schifferstein, Boelma, \& Lensvelt-Mulders, 2008), which may vary far more than that for the visual domain. One manifestation of such a difference would be the greater novelty of focussing on smell in certain individuals unfamiliar with this modality. As unfamiliar smells are often disliked (e.g., Rabin \& Cain, 1984), this would presumably diminish modality-based differences.

In the Introduction, we suggested that if something is liked more, it may also be desired more, and as the olfactory channel is argued to generate more affect, it may also generate more desire. However, prior evidence for the claim that olfaction generates more affect than vision per se is not robust. One major problem has been stimulus matching between modalities. A failure to match generates interpretive difficulties, especially since the same chemical stimulus (e.g., parmesan cheese [isovaleric acid] or pine needles [pinene]) can have different affective valence dependent upon the odor name provided by the experimenter (e.g., Herz \& von Clef, 2001). In the absence of a name, and under the unusual condition of blind smelling, many participants are not able to identify even common odorants (e.g., Cain, 1979). In the current experiment, the stimuli-snack foods-were fully counterbalanced across participants to ensure complete overall matching of visual and olfactory stimuli. To eliminate misidentification, all stimuli were accompanied by their name. Under these conditions, we obtained evidence of greater desire via olfaction. Just as we argued in the Introduction that greater liking for a smell might translate into greater desire, we suggest that the reverse also holds true, and that our greater desire findings support the notion that olfaction is a more affect-laden sense than vision.

Of interest was the observation that when hungry, foods that had been just sniffed were reported as being liked and desired more when they were later seen and eaten, relative to foods that were initially just seen. One way to conceptualize this finding is as a form of repetition priming (Smeets \& Dijksterhuis, 2014), whereby prior experience of the stimulus facilitates later processing. One interpretive issue here is that participants were clearly aware of the 'prime'-looking at or smelling the snack; however, consciously experiencing a prime does not preclude a priming effect. What is argued to be important is only that the prime's later influence is outside of awareness (e.g., Bargh, 1992). As we noted earlier, participants did not indicate any knowledge of the study aims, and so would have no reason to suspect any later influence to occur. However, as procedural awareness is difficult to exclude and debriefing was not extensive, this remains a tentative conclusion (Newell \& Shanks, 2014). Mechanistically, the putative priming effect may manifest via the initial affective reaction to the snack being reaccessed when it is later eaten (or presumably explicitly remembered if participants were procedurally aware). Thus, greater affect for sniffed snacks should result in correspondingly greater affect when the same snacks are eaten (and with correspondingly lower affective reactions for visually experienced snacks).

Apart from our data suggesting that smelling a food after a meal may be more likely to stimulate appetite than just looking at it, these findings also imply that the olfactory and visual channels of food perception are not motivationally equivalent, as many models presume (e.g., Berridge, 1996; Cabanac, 1992; Jones et al., 2018; Kavanagh et al., 2005). One way to account for this nonequivalence is to look for differences in information processing steps between the senses. In this regard, a key difference may be reliance upon memory. Visual-based judgments of desire must rely primarily on memory (i.e., recollection of prior experience with that food). However, although olfactory-based desire also utilizes memory, it alongside gustation has access to the molecular features of the stimulus, which can result in hard-wired affective reactions (e.g., Haddad, Medhanie, Roth, Harel, \& Sobel, 2010; Khan et al., 2007). If satiation affects hard-wired affective reactions in a different manner to memory-based recollections, this could potentially account for motivational nonequivalence between vision and olfaction.

In conclusion, we find that under conditions of cue equivalence, olfactory judgments led to greater desire irrespective of physiological state, and when hungry may have primed liking and desire for more evaluations made when the snack was actually consumed. Following a change in physiological state, visually based desire changed far more than olfactory-based desire, relative to liking and desire for more ratings made when the snacks were actually consumed. This all suggests that the assumption of motivational equivalence does not hold for olfaction and vision under the conditions tested in this experiment.

Acknowledgments The authors thank Alysia Robertson for assistance with these experiments and the Australian Research Council for their support (DP150100104). 


\section{References}

Attuquayefio, T., Stevenson, R. J., Boakes, R. A., Oaten, M., Yeomans, M., Mahmut, M., \& Francis, H. M. (2016). Increased consumption of a Western-style diet is associated with poorer inhibition of wanting for palatable snack foods when sated. Journal of Experimental Psychology: Animal Learning and Cognition, 42, 415-428.

Bargh, J. (1992). Why subliminality does not matter to social psychology: Awareness of the stimulus versus awareness of its effects. In R. Bornstein \& T. Pittman (Eds.), Perception without awareness: Cognitive, clinical \& social psychology (pp. 236-255). New York, NY: Guilford Press.

Berridge, K. (1996). Food reward: Brain substrates of wanting and liking. Neuroscience and Biobehavioral Reviews, 20, 1-25.

Cabanac, M. (1979). Sensory pleasure. Quarterly Review of Biology, 54 , $1-28$.

Cabanac, M. (1992). Pleasure: The common currency. Journal of Theoretical Biology, 155, 265-293.

Cain, W. (1979). To know with the nose: Keys to odor identification. Science, 203, 464-470.

Croy, I., Buschhuter, D., Seo, H.-S., Negoias, S., \& Hummel, T. (2010). Individual significance of olfaction: Development of a questionnaire. European Archives of Otolaryngology, 267, 67-71.

Dubois, D. (2000). Categories as acts of meaning: The case of categories in olfaction and audition. Cognitive Science Quarterly, 1, 35-68.

Duclaux, R., Feisthauer, J., \& Cabanac, M. (1973). Effts du repas sur l'agreement d'odeurs alimentaires at nonalimentaires chez l'homme. Physiology \& Behavior, 10, 1029-1033.

Engen, T. (1982). The perception of odors. New York, NY: Academic Press.

Ehrlichman, H., \& Bastone, L. (1992). Olfaction and emotion. In M. Serby \& K. Chobor (Eds.), Science of olfaction (pp. 410-438). New York, NT: Springer-Verlag.

Haddad, R., Medhanie, A., Roth, Y., Harel, D., \& Sobel, N. (2010). Predicting odor pleasantness with an electronic nose. PLOS Computational Biology, 6, 4.

Herz, R. (2004). A naturalistic analysis of autobiographical memories triggered by olfactory, visual, and auditory stimuli. Chemical Senses, 29, 217-224.

Herz, R., \& von Clef, J. (2001). The influence of verbal labelling on the perception of odors: Evidence for olfactory illusions. Perception, 30, 381-391.

Hinton, P., \& Henley, T. (1993). Cognitive and affective components of stimuli presented in three modes. Bulletin of the Psychonomic Society, 31, 595-598.

Hofmann, W., \& Van Dillen, L. (2012). Desire: The new hot spot in selfcontrol research. Current Directions in Psychological Science, 21, $317-322$.

Jiang, T., Soussignan, R., Rigaud, D., Martin, S., Royet, J.-P., Brondel, L., \& Schaal, B. (2008). Alliesthesia to food cues: Heterogeneity across stimuli and sensory modalities. Physiology \& Behavior, 95, 464-470.

Jones, A., Robinson, E., Duckworth, J., Kersbergen, I., Clarke, N., \& Field, M. (2018). The effects of exposure to appetitive cues on inhibitory control: A meta-analytic investigation. Appetite, 128, 271-282.

Kavanagh, D., Andrade, J., \& May, J. (2005). Imaginary relish and exquisite torture: The elaborated intrusion theory of desire. Psychological Review, 112, 446-467.

Khan, R., Luk, C., Flinker, A., Aggarwal, A., Haddad, R., \& Sobel, N. (2007). Predicting odor pleasantness from odorant structure: Pleasantness as a reflection of the physical world. Journal of Neuroscience, 27, 10015-10023.
Newell, B. \& Shanks, D. (2014). Unconscious influences on decision making: A critical review. Behavioral and Brain Sciences, 37, 1-18.

Pender, S., Stevenson, R. J., Francis, H., \& Oaten, M. (2019). Wanting and liking for sugar sweetened beverages and snacks differ following depletion and repletion with energy and fluids. Appetite, 137, $81-89$.

Rabin, M. D. \& Cain, W. S. (1984). Odor recognition: Familiarity, identifiability and encoding consistency. Journal of Experimental Psychology: Learning, Memory and Cognition, 10, 316-325.

Redden, J., \& Haws, K. (2013). Healthy satiation: The role of decreasing desire in effective self-control. Journal of Consumer Research, 39, $1100-1114$.

Richardson, J., \& Zucco, G. M (1989). Cognition and olfaction: A review. Psychological Bulletin, 105, 352-360.

Rolls, B., Rolls, E., Rowe, E., \& Sweeney, K. (1981). Sensory specific satiety in man. Physiology and Behavior, 27, 137-142.

Rouby, C., \& Bensafi, M. (2002). Is there a hedonic dimension to odors? In C. Rouby, B. Schall, D. Dubois, R. Gervais, \& A. Holley (Eds.), Olfaction, taste and cognition (pp. 140-159). Cambridge, UK: Cambridge University Press.

Royet, J.-P., Zald, D., Versace, R., Costes, N., Lavenne, F., Koenig, O., \& Gervais, R. (2000). Emotional responses to pleasant and unpleasant olfactory, visual, and auditory stimuli: A PET study. Journal of Neuroscience, 20, 7752-7759.

Russell, J. (2003). Core affect and the psychological construction of emotion. Psychological Review, 110, 145-172.

Smeets, M., \& Dijksterhuis, G. (2014). Smelly primes - when olfactory primes do or do not work. Frontiers in Psychology, 5, 96.

Smeets, M., Schifferstein, H., Boelma, S. \& Lensvelt-Mulders, G., (2008). The odour awareness scale (OAS): a new scale for measuring positive and negative odour awareness. Chemical Senses, 33, 725-734.

Stevenson, R. J. (2009). The psychology of flavour. Oxford, UK: Oxford University Press.

Stevenson, R. J., Francis, H. M., Attuquayefio, T., Gupta, D., Yeomans, M. R., Oaten, M. J., \& Davidson, T. (2020). Hippocampaldependent appetitive control is impaired by experimental exposure to a Western-style diet. Royal Society Open Science, 7, 191338.

Stevenson, R. J., Francis, H. M., Attuquayefio, T., \& Ockert, C. (2017). Explicit wanting and liking for palatable snacks are differentially affected by change in physiological state, and differentially related to salivation and hunger. Physiology and Behavior, 182, 101-106.

Wrzesniewski, A., McCauley, C., \& Rozin, P. (1999). Odor and affect: Individual differences in the impact of odor on liking for places, things and people. Chemical Senses, 24, 713-721.

Zatorre, R. (2002). Processing of olfactory affective information: Contribution of functional imaging studies. In C. Rouby, B. Schall, D. Dubois, R. Gervais, \& A. Holley (Eds.), Olfaction, taste and cognition (pp. 324-334). Cambridge, UK: Cambridge University Press.

Zuniga, A., Stevenson, R. J., Thayer, Z., Miller, L., Francis, H., Saluja, S., $\&$ Nikpour, A. (2020). The impact of hippocampal damage on appetitive control. Neurocase, 26, 305-312.

The data and materials for the experiment reported here are available from the communicating author on request, and the experiment was not preregistered.

Publisher's note Springer Nature remains neutral with regard to jurisdictional claims in published maps and institutional affiliations. 